\title{
A New Algorithm Based on Differential Transform Method for Solving Partial Differential Equation System with Initial and Boundary Conditions
}

\author{
Chenlu Huang, Jiwei Li*, Fali Lin \\ School of Architecture and Civil Engineering, Taizhou University, Taizhou, China \\ Email: ^iamliji_007@126.com
}

How to cite this paper: Huang, C.L., Li, J.W. and Lin, F.L. (2020) A New Algorithm Based on Differential Transform Method for Solving Partial Differential Equation System with Initial and Boundary Conditions. Advances in Pure Mathematics, 10, 337349.

https://doi.org/10.4236/apm.2020.105020

Received: April 25, 2020

Accepted: May 22, 2020

Published: May 25, 2020

Copyright $\odot 2020$ by author(s) and Scientific Research Publishing Inc. This work is licensed under the Creative Commons Attribution International License (CC BY 4.0).

http://creativecommons.org/licenses/by/4.0/

\begin{abstract}
In this paper, a new Fourier-differential transform method (FDTM) based on differential transformation method (DTM) is proposed. The method can effectively and quickly solve linear and nonlinear partial differential equations with initial boundary value (IBVP). According to boundary condition, the initial condition is expanded into a Fourier series. After that, the IBVP is transformed to an iterative relation in K-domain. The series solution or exact solution can be obtained. The rationality and practicability of the algorithm FDTM are verified by comparisons of the results obtained by FDTM and the existing analytical solutions.
\end{abstract}

\section{Keywords}

Differential Transform Method, Initial Boundary Value Problem, Fourier-Differential Transform Method, Fourier Series, Typical Zero Boundary

\section{Introduction}

The differential transform method (DTM) is a powerful approximate analytic method for solving linear and nonlinear differential equations. Thus it provides widely applicable technique to construct an analytical solution of differential equations in a polynomial form. Since the basic idea of the DTM was introduced by Pukhov [1], the method has been studied intensely and has substantially grown. A large amount of literature about the DTM, its applications and its extensions is available, e.g. [2]-[28]. 
Although being powerful, there still exist some difficulties in solving system of differential equations with initial and boundary condition(s) by the DTM. In fact, one may note that much of the research on the method is to solve the linear and nonlinear ODE with or without initial or boundary condition(s) [3]-[13]. Although there are some researches on solution of BVP, the BVP is composed of single equation and special initial boundary data [14] [15] [16] [17] [18] [24]. The research on solution of the initial boundary value problem of partial differential equation system based on DTM is scarce. It is well known that a PDE (a system of PDEs) cannot model any real process without additional condition(s) on the unknown function(s). On the other hand, boundary-value problems (BVPs) based on the relevant PDEs (systems), which reflect general physical laws, describe many real processes arising in nature and society.

The aim of this paper is to extend the differential transformation method to solve partial differential equation system with initial and three typical zero boundary conditions. According to the characteristics of boundary conditions, the initial function is respectively expanded into corresponding Fourier series. The method based on the iteration procedure of DTM and Fourier expansion technology is called Fourier-differential transform method (FDTM). The method can be used to evaluate the approximating solution by the finite Taylor series and by an iteration procedure described by the transformed equations obtained from the original equation using the operations of differential transformation.

\section{Differential Transform Method}

\subsection{One-Dimensional Differential Transform}

The definitions and operations of one-dimensional differential transform method (DTM) are introduced in [3]-[13]. The basic definitions of the DTM are given as follows:

The differential transform of the $k^{\text {th }}$ differentiable function $u(x)$ at $x=0$ is defined by

$$
U(k)=\left.\frac{1}{k !} \frac{\partial^{k} u(x)}{\partial x^{k}}\right|_{x=0}
$$

where $u(x)$ is the original function and $U(k)$ is the transformed function. The corresponding inverse transform is defined by

$$
u(x)=\sum_{k=0}^{\infty} U(k) x^{k}
$$

Substituting Equation (2) into Equation (1), it can be obtained as

$$
u(x)=\left.\sum_{k=0}^{\infty} \frac{1}{k !} \frac{\partial^{k} u(x)}{\partial x^{k}}\right|_{x=0} x^{k}
$$

Using the differential transform, a differential equation in the domain of interest can be transformed to be an algebraic equation in the $K$-domain and $u(x)$ 
can be obtained by finite-term Taylor series plus a remainder,

$$
u(x)=\left.\sum_{k=0}^{n} \frac{1}{k !} \frac{\partial^{k} u(x)}{\partial x^{k}}\right|_{x=0} x^{k}+R_{n+1}(x)
$$

Some of the fundamental mathematical operations performed by one-dimensional differential transform can be obtained in Table 1 .

\subsection{Two-Dimensional Differential Transform}

Consider a function of two variables $u(x, t): R \times R \rightarrow R$, based on the properties of one-dimensional DTM, if $u(x, t)$ is analytic and continuously differentiable with respect to variables in the domain of interest, the two-dimensional transform at $(0,0)$ are given as follows [18] [20] [22] [24]

$$
U(i, j)=\left.\frac{1}{i ! j !} \frac{\partial^{i+j} u(x, t)}{\partial x^{i} \partial t^{j}}\right|_{(0,0)}
$$

The spectrum function $U(i, j)$ is the transformed function, which is also called the T-function. The function $u(x, t)$ is the original function. The differential inverse transform of $U(i, j)$ is defined as follows.

$$
u(x, t)=\sum_{i=0}^{\infty} \sum_{j=0}^{\infty} U(i, j) x^{i} t^{j}
$$

Substituting Equation (6) into Equation (5), it can be obtained as

$$
u(x, t)=\left.\sum_{i=0}^{\infty} \sum_{j=0}^{\infty} \frac{1}{i ! j !} \frac{\partial^{i+j} u(x, t)}{\partial x^{i} \partial t^{j}}\right|_{(0,0)} x^{i} t^{j}
$$

Unlike the traditional high-order Taylor series method which requires complicated symbolic computations, with this method, the given equation and related conditions are transformed into a recurrence relation, through which one can easily obtain the coefficients of a Taylor series solution.

The main advantage of the DTM is that it provides an explicit and numerical solution with minimal calculations. Another important advantage is that this method can be applied directly to nonlinear problems without linearization, discretization or perturbation. Some of the fundamental mathematical operations performed by two-dimensional differential transform can be obtained in Table 2.

Table 1. One-dimensional differential transform.

\begin{tabular}{cc}
\hline Original function & Transformed function \\
\hline$u(x)=u_{1}(x) \pm u_{2}(x)$ & $U(k)=U_{1}(k) \pm U_{2}(k)$ \\
$u(x)=c v(x)$ & $U(k)=c V(k)$ \\
$u(x)=u_{1}(x) u_{2}(x)$ & $U(k)=\sum_{m=0}^{k} U_{1}(m) U_{2}(k-m)$ \\
$u(x)=\frac{\partial^{k} v(x)}{\partial x^{k}}$ & $U(k)=\frac{(k+m) !}{k !} V(k+m)$ \\
\hline
\end{tabular}


Table 2. Two-dimensional differential transform.

\begin{tabular}{cc}
\hline Original function & Transformed function \\
\hline$u(x, t)=u_{1}(x, t) \pm u_{2}(x, t)$ & $U(i, j)=U_{1}(i, j) \pm U_{2}(i, j)$ \\
$u(x, t)=c v(x, t)$ & $U(i, j)=c V(i, j)$ \\
$u(x, t)=u_{1}(x, t) u_{2}(x, t)$ & $U(i, j)=\sum_{i=0}^{m} \sum_{j=0}^{n} U_{1}(i, n-j) U_{2}(m-i, j)$ \\
$u(x, t)=\frac{\partial v(x, t)}{\partial t}$ & $U(i, j)=(j+1) V(i, j+1)$ \\
$u(x, t)=\frac{\partial^{k} v(x, t)}{\partial x^{k}}$ & $U(i, j)=\frac{(i+k) !}{k !} V(i+k, j)$ \\
$u(x, t)=\frac{\partial^{k} v(x, t)}{\partial t^{k}}$ & $U(i, j)=\frac{(j+k) !}{k !} V(i, j+k)$ \\
\end{tabular}

\section{The Fourier-Differential Transform Method (FDTM)}

Many engineering phenomenon are mathematically modeled by categorizing them in the initial boundary value problems (IBVPs). In this study, an efficient algorithm based upon the differential transform method (DTM) is considered to solve the system of partial differential equations with initial and zero boundary conditions. In order to illustrate the algorithm, a more general form of equations at $x \in[a, b], a, b \in R$ and $t \in[0, \infty]$ is expressed as

$$
\begin{aligned}
& L_{t}(u(x, t))+L_{x}(u, v)+N_{1}(u, v)=0 \\
& L_{t}(v(x, t))+L_{x}(u, v)+N_{2}(u, v)=0
\end{aligned}
$$

with initial conditions

$$
\begin{aligned}
& u(x, 0)=f(x), a<x<b \\
& v(x, 0)=g(x), a<x<b
\end{aligned}
$$

and zero Dirichlet boundary conditions

$$
\begin{aligned}
& u(a, t)=u(b, t)=0, t>0 \\
& v(a, t)=v(b, t)=0, t>0
\end{aligned}
$$

or zero Neumann boundary conditions

$$
\begin{gathered}
\left.\frac{\partial u}{\partial x}\right|_{x=a}=\left.\frac{\partial u}{\partial x}\right|_{x=b}=0, t>0 \\
\left.\frac{\partial v}{\partial x}\right|_{x=a}=\left.\frac{\partial v}{\partial x}\right|_{x=b}=0, t>0
\end{gathered}
$$

or zero mixed boundary conditions

$$
\begin{aligned}
& u(a, t)=\left.\frac{\partial u}{\partial x}\right|_{x=b}=0, t>0 \\
& v(a, t)=\left.\frac{\partial v}{\partial x}\right|_{x=b}=0, t>0
\end{aligned}
$$


where, $L$ is liner differential operators, $N$ is nonliner differential operators, the vector of main unknown functions, $x$ is spatial variable and $t$ is time.

On the basis of the DTM, we shall introduce an effective algorithm to obtain the approximate solution of Equation (8) under the initial conditions Equation (9) and the boundary conditions Equations (12)-(17). The main steps of the algorithm are:

Step 1, Appling the differential transformation in Equation (8), and an iterative formula is obtained.

$$
\begin{aligned}
& F(U(i, j), V(i, j))=0 \\
& G(U(i, j), V(i, j))=0
\end{aligned}
$$

where, $i, j=0,1,2, \cdots, N-n$.

Step 2, Duce $U(i, j)$ and $V(i, j)(i, j=n, n+1, \cdots, N)$. According to the characteristics of boundary conditions, the initial function is respectively expanded into corresponding Fourier series [29] [30]. The expanded form of the initial conditions see Table 3.

Step 3, Imposing the truncated series solution obtained in Step 2 on the initial and boundary conditions, a linear or nonlinear algebraic equations system can be obtained by

$$
\begin{gathered}
g_{1}(U(i, j), V(i, j))=0 \\
\vdots \\
g_{n}(U(i, j), V(i, j))=0
\end{gathered}
$$

where, $i, j=0,1,2, \cdots, n-1$.

Step 4, Solve system in Step3 to determine $U(i, j)$ and $V(i, j)$, and substitute the result into the truncated series solution obtained in Step 3 to obtain the final result.

Table 3. The corresponding Fourier series of initial function to boundary conditions.

\begin{tabular}{ccc}
\hline Boundary conditions & The corresponding Fourier series of initial function & Coefficient value \\
\hline $\begin{array}{c}\text { zero Dirichlet boundary } \\
\text { conditions }\end{array}$ & $f(x)=\sum_{n=0}^{\infty} A_{n} \sin \left(\frac{n \pi(x-a)}{b-a}\right)$ & $A_{n}=\frac{2}{b-a} \int_{0}^{b-a} f(x) \sin \left(\frac{n \pi(x-a)}{b-a}\right) \mathrm{d} x$ \\
$g(x)=\sum_{n=0}^{\infty} \tilde{A}_{n} \sin \left(\frac{n \pi(x-a)}{b-a}\right)$ & $\tilde{A}_{n}=\frac{2}{b-a} \int_{0}^{b-a} g(x) \sin \left(\frac{n \pi(x-a)}{b-a}\right) \mathrm{d} x$ \\
$\begin{array}{c}\text { zero Neumann boundary } \\
\text { conditions }\end{array}$ & $f(x)=B_{0}+\sum_{n=1}^{\infty} B_{n} \cos \left(\frac{n \pi(x-a)}{b-a}\right)$ & $B_{n}=\frac{2}{b-a} \int_{0}^{b-a} f(x) \cos \left(\frac{n \pi(x-a)}{b-a}\right) \mathrm{d} x$ \\
& $g(x)=\tilde{B}_{0}+\sum_{n=1}^{\infty} \tilde{B}_{n} \cos \left(\frac{n \pi(x-a)}{b-a}\right)$ & $\tilde{B}_{n}=\frac{2}{b-a} \int_{0}^{b-a} g(x) \cos \left(\frac{n \pi(x-a)}{b-a}\right) \mathrm{d} x$ \\
$\begin{array}{c}\text { zero mixed boundary } \\
\text { conditions }\end{array}$ & $g(x)=\sum_{n=0}^{\infty} \tilde{C}_{n} \sin \left(\frac{(n+1 / 2) \pi(x-a)}{2(b-a)}\right)$ & $C_{n}=\frac{2}{b-a} \int_{0}^{b-a} f(x) \sin \left(\frac{(n+1 / 2) \pi(x-a)}{b-a}\right) \mathrm{d} x$ \\
\end{tabular}




\section{Expmple}

In order to verify the effectiveness of the above method, three typical initial boundary value problems are solved. It should be noted that the following examples only serve as illustrations and the more complex problems can be tackled by the proposed technique, e.g. higher-ordered ODEs, PDEs (system) or other functional equations involving nonlinear terms with zero boundary conditions.

Example 1. The linear heat equation was first considered at $x \in[0,1]$ and $t \in[0, \infty][31]$.

$$
u_{t}=\alpha u_{x x}
$$

with the initial data

$$
u(x, 0)=1, \quad x \in(0,1)
$$

and subject to the boundary conditions

$$
\begin{aligned}
& u(0, t)=0, t>0 \\
& u(1, t)=0, t>0
\end{aligned}
$$

where, $\alpha>0$ is constant.

Based on the FDTM, we have ones

$$
U(i, j+1)=\frac{\alpha(i+1)(i+2)}{j+1} U(i+2, j)
$$

For the initial condition, it can be obtained as

$$
\sum_{i=0}^{\infty} U(i, 0) x^{i}=\frac{4}{\pi} \sum_{n=0}^{\infty} \frac{1}{2 n+1} \sum_{k=0}^{\infty} \frac{(-1)^{k}}{(2 k+1) !}((2 n+1) \pi x)^{2 k+1}
$$

which implies

$$
U(i, 0)=\frac{4}{\pi} \sum_{n=0}^{\infty} \frac{1}{2 n+1} \frac{(-1)^{\frac{i-1}{2}}}{i !}\left(\frac{(2 n+1) \pi}{2}\right)^{i}, i=1,3,5, \cdots
$$

From the boundary conditions, we have

$$
\begin{gathered}
\sum_{j=0}^{\infty} U(0, j) t^{j}=0 \\
\sum_{j=0}^{\infty} \sum_{i=0}^{\infty} U(i, j) t^{j}=0
\end{gathered}
$$

which implies

$$
\begin{gathered}
U(0, j)=0 \\
\sum_{i=0}^{\infty} U(i, j)=0
\end{gathered}
$$

Substituting Equation (27), Equation (28) and Equation (30) into Equation (25), one has

$$
U(i, j)=\sum_{n=0}^{\infty} \frac{\alpha^{j}}{j !} \frac{4}{(2 n+1) \pi} \frac{(-1)^{\frac{i-1+2 j}{2}}}{i !}((2 n+1) \pi)^{i+2 j}, \quad i=1,3,5, \cdots
$$




$$
U(i, j)=0, \quad i=0,2,4, \cdots
$$

From the definition of inverse differential transform, we get

$$
\begin{aligned}
u(x, t) & =\frac{4}{\pi} \sum_{n=0}^{\infty} \sum_{i=0}^{\infty} \frac{1}{2 n+1} \frac{(-1)^{i}}{(2 i+1) !}((2 n+1) \pi)^{2 i+1} x^{2 i+1} \sum_{j=0}^{\infty}(-1)^{j} \frac{\alpha^{j}}{j !}((2 n+1) \pi)^{2 j} t^{j} \\
& =\frac{4}{\pi} \sum_{n=0}^{\infty} \frac{1}{2 n+1} \sin ((2 n+1) \pi x) \exp \left(-((2 n+1) \pi)^{2} \alpha t\right)
\end{aligned}
$$

Note that Equation (2) is often used to describe the law of heat conduction. Because its heat conduction coefficient is constant, it belongs to the category of linear partial differential equations. For linear partial differential equations, the separation variable method is often used to solve, but this paper gives different solutions method. The solution obtained by the FDTM converge to the solution by separation of variables method [31]. This also confirms the rationality of this method.

Example 2. Partial differential equations with constant coefficients are considered in this example.

$$
\begin{gathered}
C_{1} \frac{\partial u}{\partial t}+\frac{\partial V}{\partial t}=\frac{\partial^{2} V}{\partial x^{2}} \\
\frac{\partial u}{\partial t}+C_{2} \frac{\partial v}{\partial t}=C_{3} \frac{\partial^{2} v}{\partial x^{2}}
\end{gathered}
$$

subject to initial conditions

$$
\begin{aligned}
& u(x, 0)=1 \text { in } 0<x \leq 1 \\
& v(x, 0)=1 \text { in } 0<x \leq 1
\end{aligned}
$$

and boundary conditions

$$
\begin{gathered}
u(0, t)=0, v(0, t)=0 \text { in } t>0 \\
\frac{\partial u(1, t)}{\partial x}=0, \frac{\partial v(1, t)}{\partial x}=0 \text { in } t>0
\end{gathered}
$$

where, $C_{1}, C_{2}, C_{3}$ are costant coefficient and $C_{1} C_{2} \neq 1, C_{3} \neq 0$.

Remark: The system described above has been widely used in geotechnical engineering [32].

Based on the FDTM, we have ones

$$
\begin{aligned}
& U(i, j+1)=\frac{C_{2}(i+1)(i+2) V(i+2, j)-C_{3}(i+1)(i+2) U(i+2, j)}{(j+1)\left(C_{1} C_{2}-1\right)} \\
& V(i, j+1)=\frac{C_{1} C_{3}(i+1)(i+2) U(i+2, j)-(i+1)(i+2) V(i+2, j)}{(j+1)\left(C_{1} C_{2}-1\right)}
\end{aligned}
$$

By the initial condition Equation (37) \& Equation (38), it can be obtained as

$$
\begin{aligned}
& \sum_{i=0}^{\infty} U(i, 0) x^{i}=\frac{4}{\pi} \sum_{n=0}^{\infty} \frac{1}{2 n+1} \sum_{k=0}^{\infty} \frac{(-1)^{k}}{(2 k+1) !}\left(\frac{(2 n+1) \pi x}{2}\right)^{2 k+1} \\
& \sum_{i=0}^{\infty} V(i, 0) x^{i}=\frac{4}{\pi} \sum_{n=0}^{\infty} \frac{1}{2 n+1} \sum_{k=0}^{\infty} \frac{(-1)^{k}}{(2 k+1) !}\left(\frac{(2 n+1) \pi x}{2}\right)^{2 k+1}
\end{aligned}
$$


which implies

$$
\begin{aligned}
& U(i, 0)=\frac{4}{\pi} \sum_{n=0}^{\infty} \frac{1}{2 n+1} \frac{(-1)^{\frac{i-1}{2}}}{i !}\left(\frac{(2 n+1) \pi}{2}\right)^{i}, i=1,3,5, \cdots \\
& V(i, 0)=\frac{4}{\pi} \sum_{n=0}^{\infty} \frac{1}{2 n+1} \frac{(-1)^{\frac{i-1}{2}}}{i !}\left(\frac{(2 n+1) \pi}{2}\right)^{i}, i=1,3,5, \cdots
\end{aligned}
$$

From the boundary conditions Equation (39) \& Equation (40), one has

$$
\left\{\begin{array}{l}
\sum_{j=0}^{\infty} U_{1}(0, j) t^{j}=0, \sum_{j=0}^{\infty} U_{2}(0, j) t^{j}=0 \\
\sum_{j=0}^{\infty} \sum_{i=0}^{\infty}(i+1) U_{1}(i, j) t^{j}=0 \\
\sum_{j=0}^{\infty} \sum_{i=0}^{\infty}(i+1) U_{2}(i, j) t^{j}=0
\end{array}\right.
$$

which implies

$$
\begin{array}{r}
U(0, j)=0, V(0, j)=0 \\
\left\{\begin{array}{l}
\sum_{i=0}^{\infty}(i+1) U(i+1, j)=0 \\
\sum_{i=0}^{\infty}(i+1) V(i+1, j)=0
\end{array}\right.
\end{array}
$$

Substituting Equation (45) \& Equation (46) and Equation (48) into Equation (41) \& Equation (42), and by recursive method, it can be get

$$
\begin{aligned}
& U(i, j)=\frac{4}{\pi} \sum_{n=0}^{\infty} \frac{1}{2 n+1} \frac{(-1)^{\frac{i-1+2 j}{2}}}{i !} \frac{a_{1}(j)}{j !\left(C_{1} C_{2}-1\right)^{j}}\left(\frac{(2 n+1) \pi}{2}\right)^{i+2 j}, i=1,3,5, \cdots \\
& V(i, j)=\frac{4}{\pi} \sum_{n=0}^{\infty} \frac{1}{2 n+1} \frac{(-1)^{\frac{i-1+2 j}{2}}}{i !} \frac{a_{2}(j)}{j !\left(C_{1} C_{2}-1\right)^{j}}\left(\frac{(2 n+1) \pi}{2}\right)^{i+2 j}, i=1,3,5, \cdots
\end{aligned}
$$

where, $a_{1}(j), a_{2}(j)$ satisfy the recursive formula

$$
\boldsymbol{a}(j+1)=\boldsymbol{A a}(j)
$$

where, $\boldsymbol{a}(j)=\left(a_{1}(j), a_{2}(j)\right)^{\mathrm{T}}, \quad \boldsymbol{A}=\left(\begin{array}{cc}-1 & C_{1} C_{3} \\ C_{2} & -C_{3}\end{array}\right), \quad a_{1}(0)=a_{2}(0)=1$, $j=0,1,2, \cdots$.

From the Equation (52), we have

$$
\begin{gathered}
a_{1}(j)=\frac{\left(\lambda_{1}+1\right)\left(C_{1} C_{3}-\lambda_{2}-1\right) \lambda_{1}^{j}-\left(\lambda_{2}+1\right)\left(C_{1} C_{3}-\lambda_{1}-1\right) \lambda_{2}^{j}}{C_{1} C_{3}\left(\lambda_{1}-\lambda_{2}\right)} \\
a_{2}(j)=\frac{\left(C_{1} C_{3}-\lambda_{2}-1\right) \lambda_{1}^{j}-\left(C_{1} C_{3}-\lambda_{1}-1\right) \lambda_{2}^{j}}{\lambda_{1}-\lambda_{2}}
\end{gathered}
$$

where $\lambda_{1}$ and $\lambda_{2}$ are the eigenvalues of the matrix $\boldsymbol{A}=\left(\begin{array}{cc}-1 & C_{1} C_{3} \\ C_{2} & -C_{3}\end{array}\right)$. 
By using Equation (41) \& Equation (42) and Equation (48) \& Equation (49), it can be obtained as

$$
\begin{gathered}
U_{1}(i, j)=0, i=0,2,4, \cdots \\
U_{2}(i, j)=0, i=0,2,4, \cdots
\end{gathered}
$$

According the differential inverse transform of the DTM, we get

$$
\begin{aligned}
& u(x, t)=\frac{4}{\pi} \sum_{n=0}^{\infty} \frac{1}{2 n+1} \sum_{i=1,3,5, \cdots} \frac{(-1)^{\frac{i-1}{2}}}{i !}\left(\frac{(2 n+1) \pi}{2} x\right)^{i} \sum_{j=0}^{\infty} \frac{a_{1}(j)}{j !}\left[\frac{-((2 n+1) \pi)^{2}}{4\left(C_{1} C_{2}-1\right)} t\right]^{j} \\
& v(x, t)=\frac{4}{\pi} \sum_{n=0}^{\infty} \frac{1}{2 n+1} \sum_{i=1,3,5, \cdots} \frac{(-1)^{\frac{i-1}{2}}}{i !}\left(\frac{(2 n+1) \pi x}{2}\right)^{i} \sum_{j=0}^{\infty} \frac{a_{2}(j)}{j !}\left[\frac{-((2 n+1) \pi)^{2}}{4\left(C_{1} C_{2}-1\right)} t\right]^{j}
\end{aligned}
$$

It shoud be noted that the series expression of a sine function is $\sin (x)=\sum_{i=1,3,5, \cdots} \frac{(-1)^{\frac{i-1}{2}}}{i !} x^{i}$, therefore the Equation (57) \& Equation (58) can be rewritten as

$$
\begin{aligned}
& u(x, t)=\frac{4}{\pi} \sum_{n=0}^{\infty} \frac{B_{1} \mathrm{e}^{A_{1} t}+B_{2} \mathrm{e}^{A_{2} t}}{2 n+1} \sin \left(\frac{(2 n+1) \pi}{2} x\right) \\
& v(x, t)=\frac{4}{\pi} \sum_{n=0}^{\infty} \frac{D_{1} \mathrm{e}^{A_{1} t}+D_{2} \mathrm{e}^{A_{2} t}}{2 n+1} \sin \left(\frac{(2 n+1) \pi}{2} x\right)
\end{aligned}
$$

where $A_{1}=\frac{-((2 n+1) \pi)^{2}}{4\left(C_{1} C_{2}-1\right)} \lambda_{1}, \quad A_{2}=\frac{-((2 n+1) \pi)^{2}}{4\left(C_{1} C_{2}-1\right)} \lambda_{2}$,

$$
\begin{aligned}
& B_{1}=\frac{\left(\lambda_{1}+1\right)\left(C_{1} C_{3}-\lambda_{2}-1\right)}{C_{1} C_{3}\left(\lambda_{1}-\lambda_{2}\right)}, B_{2}=\frac{-\left(\lambda_{2}+1\right)\left(C_{1} C_{3}-\lambda_{1}-1\right)}{C_{1} C_{3}\left(\lambda_{1}-\lambda_{2}\right)}, D_{1}=\frac{C_{1} C_{3}-\lambda_{2}-1}{\lambda_{1}-\lambda_{2}}, \\
& D_{2}=\frac{-\left(C_{1} C_{3}-\lambda_{1}-1\right)}{\lambda_{1}-\lambda_{2}} .
\end{aligned}
$$

Example 3. Consider the nonlinear system: coupled Burgers' equations at the interval $x \in[0, \pi]$ and $t \in[0, \infty]$.

$$
\begin{gathered}
u_{t}-u_{x x}-2 u u_{x}+(u v)_{x}=0 \\
v_{t}-v_{x x}-2 v v_{x}+(u v)_{x}=0
\end{gathered}
$$

with the initial condition

$$
\begin{aligned}
& u(0, x)=\sin (x) \\
& v(0, x)=\sin (x)
\end{aligned}
$$

and the zero Dirichlet boundary conditions

$$
\begin{aligned}
& u(t, 0)=0, \quad u(t, \pi)=0 \\
& v(t, 0)=0, \quad v(t, \pi)=0
\end{aligned}
$$

From the FDTM, it can be obtained as 


$$
\begin{aligned}
& (j+1) U(i, j+1)-(i+1)(i+2) U(i+2, j) \\
& -2 \sum_{r=0}^{i} \sum_{s=0}^{j} U(r, j-s)(i-r+1) U(i-r+1, s) \\
& +\sum_{r=0}^{i} \sum_{s=0}^{j}(r+1) U(r+1, j-s) V(i-r, s) \\
& +\sum_{r=0}^{i} \sum_{s=0}^{j}(r+1) V(r+1, j-s) U(i-r, s)=0 \\
& (j+1) V(i, j+1)-(i+1)(i+2) V(i+2, j) \\
& -2 \sum_{r=0}^{i} \sum_{s=0}^{j} V(r, j-s)(i-r+1) V(i-r+1, s) \\
& +\sum_{r=0}^{i} \sum_{s=0}^{j}(r+1) U(r+1, j-s) V(i-r, s) \\
& +\sum_{r=0}^{i} \sum_{s=0}^{j}(r+1) V(r+1, j-s) U(i-r, s)=0
\end{aligned}
$$

For the initial conditions, we get

$$
\begin{aligned}
& U(i, 0)= \begin{cases}(-1)^{\frac{i-1}{2}} \frac{1}{i !}, & i \in \text { odd } \\
0, & i \in \text { even }\end{cases} \\
& V(i, 0)= \begin{cases}(-1)^{\frac{i-1}{2}} \frac{1}{i !}, & i \in \text { odd } \\
0, & i \in \text { even }\end{cases}
\end{aligned}
$$

For the boundary conditions, we get

$$
\begin{aligned}
& U(0, j)=0, \quad \sum_{i=0}^{\infty} U(i, j)=0 \\
& V(0, j)=0, \quad \sum_{i=0}^{\infty} V(i, j)=0
\end{aligned}
$$

Then, one has

$$
\begin{aligned}
& U(i, j)= \begin{cases}\frac{(-1)^{\frac{i-1}{2}}}{i !} \frac{(-1)^{j}}{j !}, & i \in \text { odd } \\
0, & i \in \text { even }\end{cases} \\
& V(i, j)= \begin{cases}\frac{(-1)^{\frac{i-1}{2}}}{i !} \frac{(-1)^{j}}{j !}, & i \in \text { odd } \\
0, & i \in \text { even }\end{cases}
\end{aligned}
$$

so the solution for the Equations (61)-(66) are

$$
\begin{aligned}
& u(x, t)=\sum_{j=0}^{\infty} \sum_{i=0}^{\infty} \frac{(-1)^{i}}{(2 i+1) !} x^{i} \frac{(-1)^{j}}{j !} t^{j} \\
& v(x, t)=\sum_{j=0}^{\infty} \sum_{i=0}^{\infty} \frac{(-1)^{i}}{(2 i+1) !} x^{i} \frac{(-1)^{j}}{j !} t^{j}
\end{aligned}
$$

i.e. 


$$
\begin{aligned}
& u(x, t)=\sin (x) \exp (-x) \\
& v(x, t)=\sin (x) \exp (-x)
\end{aligned}
$$

\section{Conclusion}

In this paper, we propose an FDTM algorithm based on the differential transformation method and Fourier expansion technology, which can solve the IBVP system with three kinds of typical zero boundary. Especially the discontinuous boundary can be effectively solved. Finally, the heat conduction equation with discontinuous boundary conditions is solved by the algorithm. The partial differential equations described dissipation law of pore pressure which widely applied in engineering are solved according to the FDTM. In addition, nonlinear coupled Burgers' equations with the zero Dirichlet boundary conditions can also be analytically solved by the method. The results are consistent with the existing analytical solutions. It shows that the algorithm is effective and reasonable. The algorithm can be extended to complex initial boundary value problems.

\section{Acknowledgements}

This research was supported by the General project of Zhejiang Natural Science Foundation No. LY19E080008 and Zhejiang Provincial Construction Department Project No. 2019K085.

\section{Conflicts of Interest}

The authors declare no conflicts of interest regarding the publication of this paper.

\section{References}

[1] Pukhov, G.E. (1982) Differential Transforms and Circuit Theory. Journal of Circuit Theory and Applications, 10, 265-276. https://doi.org/10.1002/cta.4490100307

[2] Zhou, J.K. (1986) Differential Transformation and Its Applications for Electrical Circuits. PhD Thesis, Huazhong University Press, Wuhan.

[3] Abdel Halim Hassan, I.H. (2008) Application to Differential Transformation Method for Solving Systems of Differential Equations. Applied Mathematical Modelling, 32, 2552-2559. https://doi.org/10.1002/cta.4490100307

[4] Abdel-Halim Hassan, I.H. (2004) Differential Transformation Technique for Solving Higher-Order Initial Value Problems. Applied Mathematics and Computation, 154, 299-311. https://doi.org/10.1016/S0096-3003(03)00708-2

[5] Jang, M.J., Chen, C.L. and Li, Y.C. (2000) On Solving the Initial Value Problems Using the Differential Transformation Method. Applied Mathematics and Computation, 115, 145-160. https://doi.org/10.1016/S0096-3003(99)00137-X

[6] Abbasov, A.R.B. (2005) The Investigation of the Transient Regimes in the Nonlinear Systems by the Generalized Classical Method. Mathematical Problems in Engineering, 5, 503-519. https://doi.org/10.1155/MPE.2005.503

[7] Chen, C.K. and Ho, S.H. (1996) Application of Differential Transformation to Eigenvalue Problems. Applied Mathematics and Computation, 79, 173-188. 
https://doi.org/10.1016/0096-3003(95)00253-7

[8] Li, X., Zhou, C. and Xu, S. (2016) A New Algorithm Based on Differential Transform Method for Solving Multi-Point Boundary Value Problems. International Journal of Computer Mathematics, 93, 981-994. https://doi.org/10.1080/00207160.2015.1012070

[9] Ertürk, V.S. and Momani, S. (2007) Comparing Numerical Methods for Solving Fourth-Order Boundary Value Problems. Applied Mathematics and Computation, 188, 1963-1968. https://doi.org/10.1016/j.amc.2006.11.075

[10] Chang, S.H. and Chang, I.L. (2008) A New Algorithm for Calculating One-Dimensional Differential Transform of Nonlinear Functions. Applied Mathematics and Computation, 195, 799-808. https://doi.org/10.1016/j.amc.2007.05.026

[11] Gokdogann, A., Merdan, M. and Yildirim, A. (2012) The Modified Algorithm for the Differential Transform Method to Solution of Genesio Systems. Communications in Nonlinear Science \& Numerical Simulation, 17, 45-51. https://doi.org/10.1016/j.cnsns.2011.03.039

[12] Momani, S. and Ertürk, V.S. (2008) Solutions of Non-Linear Oscillators by the Modified Differential Transform Method. Computers and Mathematics with Applications, 55, 833-842. https://doi.org/10.1016/j.camwa.2007.05.009

[13] Chen, C.K. and Ho, S.H. (1999) Solving Partial Differential Equations by Two Dimensional Differential Transform. Applied Mathematics and Computation, 106, 171 179. https://doi.org/10.1016/S0096-3003(98)10115-7

[14] Fatoorehchi, H. and Abolghasemi, H. (2012) Computation of Analytical Laplace Transforms by the Differential Transform Method. Mathematical and Computer Modelling, 56, 145-151. https://doi.org/10.1016/j.mcm.2011.11.063

[15] Fatoorehchi, H. and Abolghasemi, H. (2013) Improving the Differential Transform Method: A Novel Technique to Obtain the Differential Transforms of Nonlinearities by the Adomian Polynomials. Applied Mathematical Modelling, 37, 6008-6017. https://doi.org/10.1016/j.apm.2012.12.007

[16] Fatoorehchi, H. and Abolghasemi, H. (2011) Differential Transform Method to Investigate Mass Transfer Phenomenon to a Falling Liquid Film System. Australian Journal of Basic and Applied Sciences, 5, 337-345.

[17] Fatoorehchi, H. and Abolghasemi, H. (2014) An Explicit Analytic Solution to the Thomas-Fermi Equation by the Improved Differential Transform Method. Acta Physica Polonica, 125, 1083-1087. https://doi.org/10.12693/APhysPolA.125.1083

[18] Kurnaz, A., Oturnac, G. and Kiris, M.E. (2005) N-Dimensional Differential Transformation Method for Solving PDEs. International Journal of Computer Mathematics, 82, 369-380. https://doi.org/10.1080/0020716042000301725

[19] Rashidi, M.M. (2009) The Modified Differential Transform Method for Solving MHD Boundary-Layer Equations. Computer Physics Communications, 180, 2210-2217. https://doi.org/10.1016/j.cpc.2009.06.029

[20] Ravi Kanth, A.S.V. and Aruna, K. (2009) Two-Dimensional Differential Transform Method for Solving Linear and Non-Linear Schrödinger Equations. Chaos Solitons Fractals, 41, 2277-2281. https://doi.org/10.1016/j.chaos.2008.08.037

[21] Yu, L.T. and Chen, C.K. (1998) The Solution of the Blasius Equation by the Differential Transformation Method. Mathematical and Computer Modelling, 28, 101-111. https://doi.org/10.1016/S0895-7177(98)00085-5

[22] Bert, C.W. (2002) Application of Differential Transform Method to Heat Conduction in Tapered Fins. Journal of Heat Transfer, 124, 208-209. 
https://doi.org/10.1115/1.1423316

[23] Jang, M.J., Chen, C.L. and Liu, Y.C. (2001) Two-Dimensional Differential Transform for Partial Differential Equations. Applied Mathematics and Computation, 121, 261-270. https://doi.org/10.1016/S0096-3003(99)00293-3

[24] Ayaz, F. (2004) Solutions of the System of Differential Equations by Differential Transform Method. Applied Mathematics and Computation, 147, 547-567. https://doi.org/10.1016/S0096-3003(02)00794-4

[25] Odibat, Z.M. (2008) Differential Transform Method for Solving Volterra Integral Equation with Separable Kernels. Mathematical and Computer Modelling, 48, 1144 1149. https://doi.org/10.1016/j.mcm.2007.12.022

[26] Odibat, Z.M., Bertelle, R., Aziz-Alaoui, R. and Duchamp, R.H.E. (2010) A Multi-Step Differential Transform Method and Application to Non-Chaotic or Chaotic Systems. Computers and Mathematics with Applications, 59, 1462-1472. https://doi.org/10.1016/j.camwa.2009.11.005

[27] Salahshour, S. and Allahviranloo, T. (2013) Application of Fuzzy Differential Transform Method for Solving Fuzzy Volterra Integral Equations. Applied Mathematical Modelling, 37, 1016-1027. https://doi.org/10.1016/j.apm.2012.03.031

[28] Suddoung, K., Charoensuk, J. and Wattanasakulpong, N. (2014) Vibration Response of Stepped FGM Beams with Elastically End Constraints Using Differential Transformation Method. Applied Acoustics, 77, 20-28. https://doi.org/10.1016/j.apacoust.2013.09.018

[29] Haberman, R. (2012) Applied Partial Differential Equations: With Fourier Series and Boundary Value Problems. Pearson Higher Education, Boston.

[30] Hesthaven, J.S., Gottlieb, S. and Gottlieb, D. (2007) Spectral Methods for TimeDependent Problems. Cambridge University Press, Cambridge. https://doi.org/10.1017/CBO9780511618352

[31] Arpaci, V. (1966) Conduction Heat Transfer. Addison-Wesley, Reading.

[32] Fredlund, D.G. and Rahardjo, H. (1993) Soil Mechanics for Unsaturated Soils. John Wiley and Sons, New York. https://doi.org/10.1002/9780470172759 